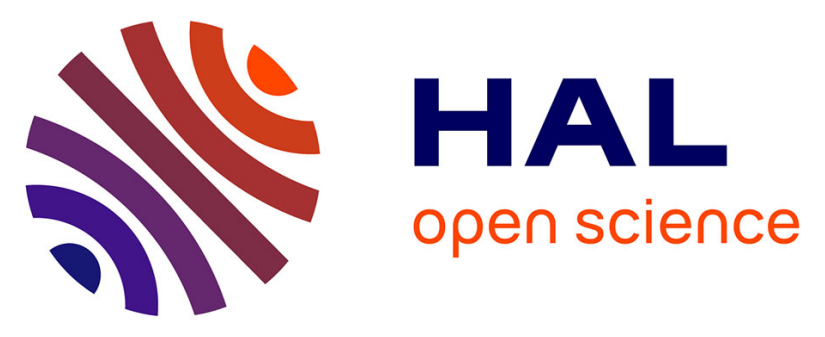

\title{
Shaping photon-pairs time-frequency correlations in inhibited-coupling hollow-core fibers
}

\author{
Martin Cordier, Adeline Orieux, Benoît Debord, Frédéric Gérôme, A. Gorse, \\ Matthieu Chafer, Eleni Diamanti, Philippe Delaye, Fetah Benabid, Isabelle \\ Zaquine
}

\section{To cite this version:}

Martin Cordier, Adeline Orieux, Benoît Debord, Frédéric Gérôme, A. Gorse, et al.. Shaping photonpairs time-frequency correlations in inhibited-coupling hollow-core fibers. Conference on Laser and Electro-Optics (CLEO: QELS Fundamental Science), May 2018, San José, CA, United States. pp.FM4G.4, 10.1364/CLEO_QELS.2018.FM4G.4 . hal-01817930

\section{HAL Id: hal-01817930 \\ https://hal-iogs.archives-ouvertes.fr/hal-01817930}

Submitted on 26 Jun 2018

HAL is a multi-disciplinary open access archive for the deposit and dissemination of scientific research documents, whether they are published or not. The documents may come from teaching and research institutions in France or abroad, or from public or private research centers.
L'archive ouverte pluridisciplinaire HAL, est destinée au dépôt et à la diffusion de documents scientifiques de niveau recherche, publiés ou non, émanant des établissements d'enseignement et de recherche français ou étrangers, des laboratoires publics ou privés. 


\title{
Shaping photon-pairs time-frequency correlations in inhibited-coupling hollow-core fibers
}

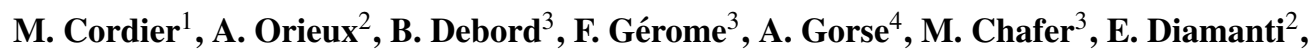 \\ P. Delaye ${ }^{5}$, F. Benabid ${ }^{3}$ and I. Zaquine ${ }^{1}$ \\ ${ }^{1}$ Laboratoire de Traitement et Communication de l'Information, Télécom ParisTech, Université Paris-Saclay, 75013 \\ Paris, France \\ ${ }^{2}$ Laboratoire d'Informatique de Paris 6, CNRS, Université Pierre et Marie Curie, Sorbonne Universités, 75005 Paris, \\ France \\ ${ }^{3}$ GPPMM Group, XLIM Research Institute, CNRS UMR 7252, University of Limoges, Limoges, France \\ ${ }^{4}$ GLOphotonics S.A.S., 123 rue Albert Thomas, Limoges, France \\ ${ }^{5}$ Laboratoire Charles Fabry, Institut dOptique Graduate School, CNRS, Université Paris-Saclay, 91127 Palaiseau \\ cedex, France \\ martin.cordier@telecom-paristech.fr and isabelle.zaquine@telecom-paristech.fr
}

\begin{abstract}
We experimentally show how multiband dispersion properties of inhibitedcoupling hollow-core fibers allow to control the spectral correlations of photon pairs generated through four-wave-mixing in a fiber filled with non-linear gas. (C) 2018 The Author(s)
\end{abstract}

OCIS codes: (060.5295) Photonic crystal fibers; (190.4380) Nonlinear optics, four-wave mixing; (270.5565) Quantum communications

Temporal modes (TM) of light are a promising resource for quantum information as they span a discrete infinite dimensional encoding basis [1]. Additionally, TM are compatible with standard fibered communication as they share the same polarization, carrier frequency and transverse spatial mode properties. Single photons in specific TM can be obtained by Spontaneous Parametric Down Conversion (SPDC) and Spontaneous Four-Wave-Mixing (SFWM) sources. Following a standard perturbative approach, the two-photon state produced by SPDC or SFWM is given by :

$$
|\psi\rangle=|0\rangle_{s}|0\rangle_{i}+\kappa \iint d \omega_{s} d \omega_{i} F\left(\omega_{s}, \omega_{i}\right)\left|\omega_{s}\right\rangle_{s}\left|\omega_{i}\right\rangle_{i}
$$

where $F\left(\omega_{s}, \omega_{i}\right)$ defined as the Joint Spectral Amplitude (JSA) is the result of energy and momentum conservation constrains. This JSA function describes the time-frequency correlations between signal and idler photons. The Schmidt decomposition of the JSA function gives the two photons decomposition in the TM basis: $F=\sum_{n} \sqrt{\lambda_{n}} S_{n}\left(\omega_{s}\right) I_{n}\left(\omega_{i}\right)$ where the $\left(\left\{S_{n}\right\}\right)$ and $\left(\left\{I_{n}\right\}\right)$ are the TM basis of the signal and idler photons. It has been shown [2] that it is possible to reduce this decomposition to one non zero term. In this case, both signal and idler photons are generated in a single TM, giving rise to a factorable state of the pair. This can be achieved if a group velocity matching condition is satisfied (either $\beta_{1}\left(\omega_{p}\right)=\beta_{1}\left(\omega_{s}\right)$ or $\beta_{1}\left(\omega_{p}\right)=\beta_{1}\left(\omega_{i}\right)$ or $2 \beta_{1}\left(\omega_{p}\right)=\beta_{1}\left(\omega_{s}\right)+\beta_{1}\left(\omega_{i}\right)$ in the case of SFWM with $\beta_{1}$ the inverse group velocity). Such single TM states are very useful for scalable heralded photon sources [3]. Here we show experimentally in a stimulated regime how this can be obtained in a Xenon-filled inhibited-coupling (IC) fiber.

IC fibers exhibit a discontinuous multiband dispersion [4] where the position of the discontinuities are dictated by the strut thickness. Such exotic dispersion opens new possibilities for the FWM phase matching fulfillment, and thus for tailoring the JSA. With the ability to have the pump and the generated signal and idler photons lying in different dispersion band, group-velocity matching can be easily obtained as shown in Figure 1. In order to demonstrate this experimentally, we use stimulated emission tomography. This technique consists of reconstructing slice by slice the JSA using a tunable continuous laser which seed the FWM. With a pump wavelength of $1030 \mathrm{~nm}$ and a seed laser at telecom wavelength, we chose an optimized fiber with a strut thickness $\mathrm{t}=600 \mathrm{~nm}$ and $40 \mu \mathrm{m}$ inner-diameter [4]. This fiber design with a discontinuity at $1.25 \mu \mathrm{m}$ in the dispersion allows a multiband FWM (i.e the idler lyes in a different band than the pump and signal). Losses have been measured around $0.02 \mathrm{~dB} / \mathrm{m}$ in the $1 \mu \mathrm{m}$ band, and $0.07 \mathrm{~dB} / \mathrm{m}$ in the $1.5 \mu \mathrm{m}$ band.

The choice of Xenon is based on two important criteria: i) being a noble gas exhibiting no Raman-Scattering which is usually the main source of noise in fibered photon-pair generation; ii) high non-linearity (in the order of $10^{-21} \mathrm{~m}^{2} / \mathrm{W}$ at 10 bar). 
(a)

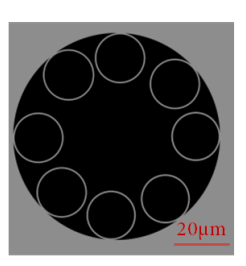

(b)
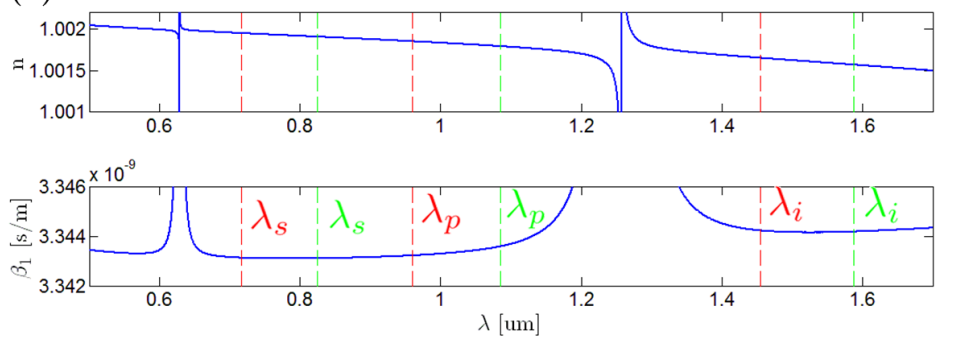

Fig. 1. (a) SEM image of the fiber cross-section (b) Calculated effective index $n_{\text {eff }}$ and inverse group velocity $\beta_{1}$ of the fundamental mode in a 8 tubes IC fiber filled with Xenon $(3 \mathrm{bar})$. The red and green dashed lines represent two specific four-wave-mixing configurations with respectively the fulfillment of $\beta_{1}\left(\omega_{p}\right)=\beta_{1}\left(\omega_{s}\right)$ and $2 \beta_{1}\left(\omega_{p}\right)=\beta_{1}\left(\omega_{s}\right)+\beta_{1}\left(\omega_{i}\right)$.

For a given fiber design, the pump wavelength, gas pressure and fiber length are three parameters which can be tuned to access various JSA shapes as illustrated in Figure 2. Both single TM (Fig2 b. and d.) or, opposingly, multi-TM (Fig2 a. and c.) can be obtained. Changing the gas pressure impacts the medium dispersion, which results in shifting the JSA and modifying its shape. This tunability can be really useful when paralleling multiple fibers (i.e each fiber being a heralding single photon source) or to aim a specific wavelength as for instance a quantum memory atomic transition. With an adequate choice of gas pressure, we experimentally obtain a FWM involving a signal at $771 \mathrm{~nm}$ and an idler at $1550 \mathrm{~nm}$.
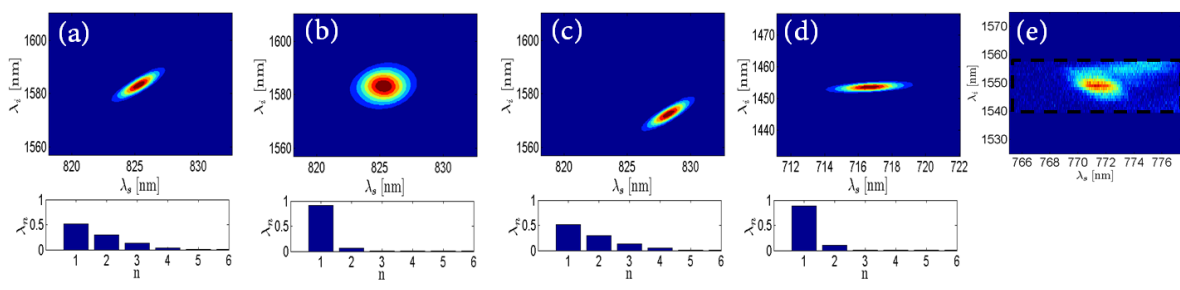

Fig. 2. Simulated (a-d) and experimental (e) JSI $\left(=|\mathrm{JSA}|^{2}\right)$ and their corresponding Schmidt decomposition for different configurations of length, gas pressure and pump wavelength. Starting from the conditions of (a) $\mathrm{P}$ $=3$ bar $\lambda_{p}=1085 \mathrm{~nm} \mathrm{~L}=1 \mathrm{~m}$, (b-d) correspond to the change of one parameter (b) $\mathrm{L}=30 \mathrm{~cm}$ (c) $\mathrm{P}=2.6$ bar (d) $\lambda_{p}=985 \mathrm{~nm}$ (e) preliminary experimental result obtained at $\lambda=1030 \mathrm{~nm}$. The black dashed rectangle shows the limits of our measurement range limitated by the seed laser tunability.

We have made a new proposal of using multi-dispersion band medium in order to obtain a real time tuning of photon-pairs time-frequency correlations. For the first time to our knowledge, we implement this technique using gasfilled inhibited-coupling fiber. Our latest experimental results on different fiber/gas configurations will be developed during the conference.

\section{References}

1. B. Brecht, D. V. Reddy, C. Silberhorn, and M. Raymer, "Photon temporal modes: a complete framework for quantum information science,"Physical Review X 5, 041017 (2015).

2. W. P. Grice, A. B. URen, and I. A. Walmsley, "Eliminating frequency and space-time correlations in multiphoton states," Physical Review A 64, 063815 (2001).

3. Meyer-Scott, Evan, et al. "Filtering is not enough for pure, efficient photon pairs." arXiv preprint arXiv:1702.05501 (2017).

4. B. Debord et al, "Ultralow transmission loss in inhibited-coupling guiding hollow fibers, “Optica, 4, 209 (2017). 